ARTICLES

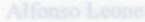

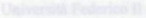

(4)

4.

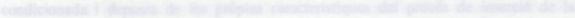
Can

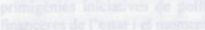

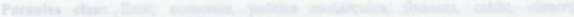

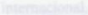




\title{
Alfonso il Magnanimo e l'economia dell'Italia meridionale
}

\author{
Alfonso Leone \\ Università Federico II \\ Dippartimento di Historia \\ (Napoles) Italia
}

\section{Resum}

La incidència de la política d'Alfons el Magnànim en fa economía, exístent, estava molt condicionada i depenia de les pròpies caracteristiques del procés de inserció de la economia del Mezzogiorno en l'estructura econòmica internacional. Mancada dels mitjans i depenent en bona mesura de factors externs, com la banca i el crèdit, les primigènies iniciatives de politica econòmica es veien interferides per les necessitats financeres de l'estat i el moment.

Paraules clau: Estat, economia, política monàrquica, finances, crèdit, comerç internacional.

Abstract. Alphonso the Magnanimous and the economy of southern Italy

The impact of Alfonso the Magnanimous' politics regarding the economy was very much a result of the particularities of the Mezzogiomo and of the international economic structure of the day. The earliest economic initiatives were impeded considerably by the financial needs of the state and the exigencies of the moment, compounded by a lack of resources and a dependence on external factors (such as banks and credit).

Key words: State, economy, monarchic politics, finances, credit, international trade.

L'influenza esercitata dalla politica o dallo stato sulla vita economica e sociale del Mezzogiorno italiano durante il Medioevo a tratti fu senza dubbio rilevante e significativa. Non credo tuttavia che si possa porre l'accento sulla iniziativa statale, come ha suggerito più volte $M$. Del Treppo', giacchè questa scelta (che altera in realtà il rapporto fra sfera

${ }^{1}$ Scrive il Del Treppo nel saggio Prospettive mediterranee nella politica economica di Federico II (in Federico II. Convegno dell'Istituto Storico Germanico di Roma nell'VIII Centenario della nascita, a cura di A. Esch e N. Kamp, Tubingen 1996, p. 338) che snel 
economica e sfera politica) porta in secondo piano il quadro storicoeconomico e traccia una visione necessariamente progressiva dell'economia e della politica. Se si considera l'età di Federico II, la penetrazione commerciale italiana, già avviatasi nella seconda metà del secolo precedente, mentre assicurò alla monarchia cospicue entrate tributarie e offri un più valido sbocco mercantile alla produzione agricola, a vantaggio soprattutto dei feudatari e proprietari terrieri, concesse ai commercianti regnicoli solo l'ambito delle attività interne e di mediazione, incidendo in misura notevole sulla struttura sociale. Gli esponenti di alcune famiglie originarie del ducato amalfitano, come i della Marra e i Pironti di Ravello, i quali si inserirono nell'ammistrazione finanziaria del Regno e anticiparono allo stato i proventi fiscali non ancora riscossi, misero a frutto le esigenze crescenti dello stato stesso. $\mathrm{Ma}$ anche il mondo rurale, specie le località e le campagne dell'entroterra - in un territorio privo in gran parte di centri cittadini, talora accidentato, suddiviso in aree definite e contraddistinto dalla tendenza all'isolamento degli insediamenti -, non potè non risentire del ruolo acquisito dal Mezzogiorno negli scambi. Prevalsero le istanze dei baroni e delle signorie monastiche, e, per esempio, l'abbazia di Cava oppose viva resistenza alla richiesta dei suoi concessionari di trasformara i censi in natura in censi in danaro. Sicchè occorre ribadire la dipendenza del mercato meridionale, assunto nel suo insieme, dal commercio internazionale, che divenne più profonda e spiccata, e delineò la fisionomia economico-sociale del Paese nei secoli successivi, pur con le sue diferente regionali. Peraltro Del Treppo avverte il rapporto del Regno con lo spazio mediterraneo, ma in chiave fortemente positiva, fondata sull'azione costruttiva del soggetto politico: ( $L$ a politica del re di Sicilia... fu anche una risposta alle sollecitazioni della congiuntura internazionale, che al Mezzogiorno chiedeva un eccezionale, e diverso rispetto al passato, incremento della sua produzione agricola, mentre ad esso assicurava nuovi sbocchi, anche e soprattutto nel bacino occidentale del Mediterraneo $)^{2}$.

D'altro canto, la stessa espressione di (politica economica) va adottata con cautela. D. Abulafia ha sottolineato opportunamente le preoccupazioni di natura finanziaria che guidarono l'imperatore svevo $^{3}$; il

\footnotetext{
Mezzogiorno, fattore di innovazione e di progresso economico fu lo Stato, ogni qual volta esso pervenne in mani forti, cosi con Federico II come con Carlo I d'Angió, come con Alfonso V d'Aragona e suo figlio Ferranter.

${ }^{2}$ Op. cit., p. 336.

${ }^{3}$ Cfr. D. ABULAFIA, Lo Stato e la vita economica, in Federico II e il mondo mediterraneo, a cura di P. Toubert e A. Paravicini Bagliani, Palermo 1994, pp. 165-187.
} 
quale, giova ricordarlo, impose un'aspra pressione fiscale, essenziale ai suoi fini politici. Forse I'idea di una politica statale diretta allo sviluppo, almeno perchè quest'ultimo sorregge lo stato stesso («quando lo Signore have li vaxalli ricchi, lui non po essere poviros), cominciò a profilarsi soltanto nei decenni di Ferrante I, con l'opera di Diomede Carafa, dove si coglie nel medesimo tempo un'analisi acuta dell'economia napoletana: $\backsim \mathrm{E} t$ questa parte de la mercantia èi cosa da delectarence bene, chè tucto di se vede paese sterilissimo, gente industriosa li fanno habundantia et se non ce creati de li vostri, lli veneranno deli foristeri et se haverano loro la utilità et se la portaranno al loro paese et case... ). Appariva insufficiente, in altre parole, il vigore delle industria e in particolare dei commerci fuori del Regno, commerci che il re aveva tentato di promuovere mediante il costoso armamento di galeazze mercantili, «dovo may in quisto regno li fo persona patronasse altro che sagecties".

I sovrani aragonesi concessero ai mercanti stranieri salvacondotti ed esenzioni daziarie, e una celebre prammatica del 4 giugno 1469 riconobbe la cittadinanza napoletana, con i conseguenti diritti, a coloro che si stabilivano nella citta e vi prendevano moglie. I provvedimenti contro i fiorentini furono dettati dalle ragioni della politica internazionale, sia quello di espulsione nel 1447, sia quello di confisca dei beni del 1478, seguito alla scomunica di Lorenzo il Magnifico da parte del pontefice Sisto IV. Tali episodi non ostacolarono però l'espansione fiorentina, che si mostrò sempre più estesa, in un periodo caratterizzato dal predominio del capitale mercantile. Si può dire dunque che la dinastia continuasse a stimare il commercio estero soprattutto come una fonte preziosa di entrate e di prestiti; e che in ultima analisi, dagli Svevi agli Aragonesi, l'unico strumento effettivo di politica economica, in quanto utile allo svolgimento dei traffici, sia stato il privilegio di fiera. A metà del Quattrocento si era consolidata una vera e propria rete di fiere, costituita da alcune di maggior respiro e prestigio, come quelle di Salerno, Gaeta, Lanciano, Trani, e da numerose altre di interesse locale: esse garantirono la circolazione delle merci e sostennero anche il credito, grazie all'usanza del pagamento differito.

Neanche le finanze statali e l'amministrazione finanziaria mettono in luce un ruolo determinante dello stato nel processo economico. Nel corso del Trecento e del Quattrocento, come nel Cinquecento, lo stato

${ }^{4}$ Cfr. L. MIELE, Modelli e ruoli sociali nei aMemorialin di Diomede Carafa, Napoli 1989, p. 44 ss.; e D. ABULAFIA. "The Crown and the economy under Ferrante I of Naples (1458-1494)", in City and Countryside in late medieval and Renaissance Italy. Essays presentes to Philip Jones, a cura di T. Dean e C. Wickham, London 1990, pp. $125-146$. 
fece fronte ai bisogni finanziari mediante vendite o appalti di uffici pubblici, vendite di terre demaniali, di giurisdizioni, di cespiti, di esazioni di imposte, di riscossioni di donativi e soprattutto prestiti e anticipi sotto forma di partiti e cambi, con tassi elevati, da parte dei mercanti-banchieri operanti nella capitale, in primo luogo fiorentini e, dopo la conquista del Magnanimo, catalani. Spesso ad anticipare il gettito annuo presunto delle imposte o le somme dei donativi erano i medesimi banchieri che erogavano di solito i prest iti. Questa prassi - se comportò in alcuni momenti un adeguamento dell'amministrazione ai progressi del servizio bancario, come avvenne negli anni ottanta del Quattrocento per il legame privilegiato della tesoreria regia con il banco Strozzi; e se potè favorire una certa area sociale, quella dei funcionari e commissari pubblici -, alimentò il controllo della banca fiorentina sulla finanza pubblica, che rappresenta un aspetto ben chiaro dell'egemonia toscana sulla realtà economica del Paese. Anche una compagnia di livello limitato quale quella di Tommaso Ginori, come ha rilevato il Cassandro, perseguì una linea, che sembra a tutte comune, di acquisizione di introiti fiscali, «come mezzo di graduale rimborso delle forti somme prestate alla regia corte $\aleph^{5}$. La preminenza finanziaria, altresì, tornava proficua agli stessi fini commerciali, tanto che il partito delle tratte si configurò come il tramite principale nella estrazione dẹ cereali e dell'olio pugliese. Nè pare escluso che essa condizionasse gli atteggiamenti e le decisioni di natura politica.

II Magnanimo introdusse probabilmente l'uso degli albarani regi, i quali potevano ottenere una proroga della scadenza o essere rinnovati con una ricontrattazione del prestito, e venivano ceduti dai beneficiari come normali titoli di credito. Gli importi, talvolta coperti da redditi determinati, erano anche considerevoli, ad esempio di diecimila ducati in un titolo rilasciato al catalano Bernardo Corbera. Del pari la corte in epoca aragonesa si avvalse di frequente della edetta pianas ossia dell'ordine verbale in banco ${ }^{6}$. Il pegno dei gioielli fu consueto dalla fine del Duecento, e le fonti quattrocentesche, al riguardo, confermano con efficacia la consistenza dell'esposizione verso i mercanti: nel 1481 , Battista Pandolfini ricevette gioielli e libri per un prestito di 38.000 ducati, dovuto alle spese della guerra; nel dicembre del 1484 Filippo Strozzi aveva in suo potere pietre preziose per un valore di circa 50.000

${ }^{5}$ Cfr. M. CASSANDRO, "Affari e uomini d'affari fiorentini a Napoli sotto Ferrante I d'Aragona (1472-1495)", in Studi di storia economica toscana nel Medioevo e nel Rinascimento in memoria di Federigo Melis, Pisa 1987, p. 112.

${ }^{6} \mathrm{Cfr}$. M. DEL TREPPO, "I Catalani a Napoli e le loro pratiche con la corte", in Studi di sloria meridionale in memoria di Pietro Laveglia. Salerno 1994, p. 71 ss. 
ducati, ma il suo credito ascendeva alla somma di ben 164.000 ducati; e Carlo Borromei e Angelo Serragli ebbero in pegno nel 1486 una pietra di pregio notevole detta «a Rocca), a parziale garanzia di un credito di centomila ducati.

La funzione dello stato, inf ine, prende risalto dalla documentazione di origine pubblica, ma quella privata, notarile e mercantile, richiama l'attenzione da un lato sulla realtà economica dei vari ambiti regionali, dall'altro lato sulle forme e sul peso decisivo del commercio internazionale?

Ora, durante l'età angioina, dopo la separazione politica ed economica della Sicilia dal Regno meridionale, si manifestarono quali tratti fondamentali del mercato napoletano la struttura della bilancia commerciale, la debolezza di un ceto medio attivo, l'esiguità delle risorse finanziarie; e si accrebbe, più visibilmente con la crisi del Trecento, la dipendenza dell'economia tutta dal commercio estero. Nel periodo della dominazione aragonesa, poi, l'inserimento del Mezzogiorno nel mercato mediterraneo si realizzò in modo più compiuto ed organico, attraverso un ruolo che scaturiva dalle sue intime caratteristiche.

L'impresa di Alfonso V congiunse di fatto il Regno con l'area politico-economica catalano-aragonese, parte importante del più vasto spazio mercantile. Il sovrano elaborò anche, alla fine del 1449, un programma articolato, teso alla spiena integrazione della produzione e dei mercati di tutti i Regni della Corona d'Aragonar", e ben consapevole dell'utilità del mercato meridionale per il commercio catalano e della tradizionale impronta agricola del Mezzogiorno. Tuttavia mi sembra che non si possa attribuire alla sua azione un rilievo particolare. Come ha osservato di recente S. Epstein, anche a proposito del progetto enunciato dal Magnanimo, é difficile supporre una strategia economica in mancanza di iniziative concrete, coerenti con il disegno proposto; e gli stati dell'epoca, comunque, non possedevano ancora gli strumenti tecnici e i mezzi amministrativi e finanziari per intervenire con successo isulle strutture economiche di fondo della società y. Qui preme accennare,

${ }^{7}$ Cfr. A. LEONE, "Le fonti documentarie per la storia economica e sociale del Regno di Napoli nell'età aragonese (1443-1501)" in Valoración del estudio de las fuentes históricas jurídicas y literarias hispanas ante el siglo XXI. Atti del Convegno (Barcellona, 12-15 giugno 1996), in corso di stampa.

${ }^{8}$ Cfr. M. DEL TREPPO, "Stranieri nel Regno di Napoli.. Le elites finanziarie e la strutturazione dello spazio economico e politico" in Dentro la città. Stranieri e realtà urbane nell'Europa dei secoli XII-XVI, a cura di G. Rossetti, Napoli 1989, pp. 205 ss.

9 Cfr. S. EPSTEIN, "Dualismo economico, pluralismo istituzionale in Italia nel Rinascimento" in Revista d'Història Medieval, 6, Valéncia 1995, p. 68 s. 
soprattutto, come la struttura serrata e unitaria del mercato internazionale implicasse una maggiore subordinazione della vita economica, e ne rendesse più chiaro lo svolgimento, e come dal contesto dei traffici commerciali e marittimi e dalla coesione finanziaria dei mercati il mercato napoletano traesse contorni più limpidi, di modo che Napoli raggiunse via via il rango di una grande piazza cambiaria e assicurativa.

La politica del Magnanimo non seguì in realtà un indirizzo protezionistico, a difesa degli interessi barcellonesi e catalani, e invece fu segnata dalle costanti necessità finanziarie. Fin dal parlamento generale di San Lorenzo del marzo 1443 il re riformò la contribuzione fiscale in senso più moderno, abolendo la (subventio generalis»), cioè la colletta, e fissando l'imposta ordinaria e regolare, il focatico, a dieci carlini per fuoco, più l'acquisto di un tomolo di sale al prezzo di mezzo ducato, da pagare in tre rate e da riscuotere mediante la numerazione triennale dei fuochi effettuata da commissari pubblici e l'apprezzo, che era la ripartizione del carico fiscale tra le famiglie eseguita dalle università. L'intento di questo sistema emerge esplicitamente da una prammatica di Ferrante del marzo 1470: «sate dunque ogni diligenza necessaria, ed opportuna che alle cose predette sia data pronta e devota esecuzione, sì che i nostri ordinari pagamenti si abbiano ne' suoi termini, onde per la cessazione di quei ne' termini dovuti non succeda, o non incorra interesse, per soddisfare al quale sia necessario per l'avvenire imporre più ai nostri popoli carichi estraordinari il che sarebbe contra il proposito di nostra mente, come avendo nostri ordinarj pagamenti in suoi tempi, di quei siamo contenti e non intendiamo oltra di quei gravare $i$ nostri popoli $)^{10}$. Ma i due re aragonesi mantennero I'(adoha), cioè il tributo militare dovuto dai possessori di feudi, e le tassazioni straordinarie in occasione dell'incoronazione e del maritaggio dei figli del re e nel caso di riscatto del sovrano dalla prigionia; caso, quest'ultimo, nel quale Alfonso tendeva ad includere tutte le circostanze riguardanti la sicurezza dello stato. Inoltre il metodo dell'apprezzo, conservato dal sistema tributario angioino, provocava le incertezze di valutazione, gli abusi e le lagnanze che si desumono tanto dalla documentazione, quanto da un'altra prammatica di Ferrante, la De appretio seu bonorum aestimatione, del novembre 1467 , la quale, peraltro, fu riconfermata per circa tre secoli. Le riscossioni e i versamenti non avevano norme inderogabili, e la revisione dei conti, compito della Camera della Sommaria, avveniva con lunghi ritardi. Quanto alle imposte indirette (dazi, gabelle, diritti, ecc.),

${ }^{10} \mathrm{Cfr}$. A. BULGARELl LUKACS, L'imposta diretta nel Regno di Napoli in eta moderna, Milano, 1993, p, 121. 
si affermò l'arrendamento, che poi fu molto diffuso nel corso del Cinquecento e del Seicento. Alfonso ricorse ripetutamente al prelievo straordinario: due tassazioni furono deliberate, per esempio, nel parlamento del 1449 e tre in quello dell'anno seguente; e nell'ottobre del '56 egli portò l'imposta diretta a venti carlini, con la distribuzione gratuita del sale. Infatti il gettito annuo del focatico, previsto di circa 400.000 ducati, era stato ben minore, dal momento che nel '49 si calcolarono 230.000 fuochi tassabili. Il re prese iniziative redditizie, come la regolamentazione della dogana di Foggia, una delle risorse più feconde dell'erario, il riscatto e la gestione diretta delle saline, il ripristino del monopolio del ferro, dell'acciaio e della pece, le inchieste del 1454 per l'avocazione al potere regio dei passi interni; e praticò anche il commercio del grano, attraverso i maestri portolani e i compratori maggiori. Ma non vi é dubbio che le entrate, pur con un fisco gravoso per il Regno - come Ferrante riconobbe nel parlamento del luglio 1458, subito dopo la morte di Alfonso -, fossero inadeguate rispetto alle spese militari e di corte; e che il credito mercantile rimanesse di rilevanza centrale. Accanto alla organizzazione statale verso forme moderne si verificò nel settore finanziario l'uso assiduo di uno strumento come la lettera di cambio, che nel mondo economico dell'epoca permetteva la circolazione internazionale del credito. Si tratta della testimonianza di una utilizzazione del cambio, offerta dagli operatori, e di un bisogno pronunciato di finanziamento e di anticipazioni, che ricadevano in larga parte su Barcellona, dove suscitavano sensibile malcontento: ma pure di un rapporto debitorio molto significativo verso i mercanti-banchieri fiorentini e catalani. La documentazione illustrata da $\mathrm{R}$. Conde descrive dal vivo quanto al sovrano stesse a cuore fruire del mercato di capitali e onorare gli obblighi contratti, nonostante non gli fosse agevole". Egli sapeva di non poter rinunciare alla spirale del credito mercantile. L'indebitamento dello stato e le ingenti uscite, in definitiva, non vanno ritenuti come il costo del riordinamento statale, e forse riordinamento statale e precarietà della finanza pubblica concorsero alla politica di potenza e di prestigio di un principe rinascimentale. E il rapporto incontrollato con gli operatori financiari e l'uso dei mezzi propri dell'attività mercantile non costituirono innanzitutto una gestione più duttile e dinamica della finanza pubblica, nell'ambito del rinnovamento dello stato, ma piuttosto il risvolto debole della compagine statale, se la crescita dell'esposizione

$"$ Cfr. R. CONDE Y DELGADO DE MOLINA. "La letra de cambio en el sistema financiero de Alfonso el Magnanimo" in XIV Congresso di storia della Corona d'Aragona, vol. III, Sassari 1996, pp. 257-269. 
era destinata a tradursi in motivo di fragilità economica e politica della monarchia. Come si é detto, nel periodo aragonese il percorso storicoeconomico del Mezzogiorno pervenne ad una fase matura, sulla quale il progresso giuridico e civile non ebbe specifica incidenza.

L'aggregazione nuova e organica nel mercato internazionale, fattosi a sua volta più compatto, ordinato e capitalistico, avvenne dopo la morte di Alfonso e il distacco del Regno dai domini della corona. I traffici dei Catalani a Napoli e nelle province e le loro strette relazioni con la corte, susseguenti alla conquista, proseguirono vivaci, pur con qualche arretramento, convivendo con il primato fiorentino; e probabilmente Napoli restò un versante forte del commercio catalano anche durante il declino di Barcellona. É un indizio evidente dell'ampiezza del mercato meridionale. Ma risultò decisiva l'attività commerciale florentina, condotta in collegamento con le compagnie impiantate fuori del Regno, spesso su commissione, e con $\mathrm{i}$ contatti finanziari esterni più convenienti. I Fiorentini importarono in grosse quantità $i$ tessuti, non soltanto di produzione toscana, e si dedicarono all'estrazione molto remunerativa del grano pugliese e della seta calabrese, aprendo a questo scopo piccole ditte bancarie e mercantili a Trani, a Lecce, a Cosenza. Intanto la loro rete bancaria, insediata nei centri principali dello scambio, era ascesa a cardine capitalistico incontrastato del commercio internazionale, e aveva ottenuto la coesistenza e l'equilibrio economico dei mercati; e attuava il coinvolgimento dell'area napoletana appunto nella connessione finanziaria dei mercati. Dalle filiali degli Strozzi e dei Medici, Napoli fu collegata sul piano finanziario con Roma, Firenze, Pisa, Milano, Venezia, Genova, Palermo, Barcellona, Valenza, Lione, Avignone, Montpellier, Ginevra, Bruges e Londra; i profitti conseguiti dal commercio estero si mutarono in una risorsa proficuamente impiegata in altre articolazioni del tessuto mercantile mediterraneo. Addirittura fu possibile reperire sulla piazza napoletana la copertura assicurativa, fornita in una certa misura dai medi operatori catalani, per il trasporto delle lane inglesi da Southampton a Porto Pisano, Piombino e Talamone.

Gli affari commerciali e le incette dei Fiorentini si svilupparono con una efficienza operativa che in effetti fu un prezioso raccordo interno, legato con le fasi dell'importazione e dell'esportazione, e le compagnie concessero di norma un credito alla clientela (anticipazioni, dilazioni di pagamento, rinnovi, mallevadorie), senza il quale questa non avrebbe sopportato lo scompenso della bilancia commerciale. In relazione al rapporto fra cominercio estero e Mezzogiorno, tutto ciò aggravò la contraddizione, o il paradosso, insita nello svolgimento della vita 
economica: che il commercio estero rafforzava la radicata subordinazione, e si rivelava sempre più come la linfa indispensabile di una società nel suo insieme priva di autonomia commerciale.

Il commercio a breve distanza fu una componente integrante del Mediterraneo occidentale: ne ha esposti alcuni aspetti Abulafia nel corso del recente XVI Congresso di storia della Corona d'Aragona, ai fini di un quadro non costruito esclusivamente intorno all'intervento statale. A quello tra Campania, Calabria e Sicilia parteciparono commercianti catalani, genovesi e pisani, sia direttamente, sia con fattori o agenti, e navi che talora percorrevano rotte più lunghe. In esso agivano anche $\mathrm{i}$ centri marittimi come Amalfi. Nondimeno tale apporto dei regnicoli, di tono subaiterno, non toccava che la fascia costiera, e non era sufficiente, per volume ed entità di capitali, a ridurre la dipendenza dell'economia dal commercio e dal finanziamento estero.

La prospettiva, per concludere, in cui intendere l'opera e la figura del Magnanimo quale re di Napoli é data dal generale avvicinamento dell'Italia meridionale al mondo iberico e mediterraneo; e questo generale avvicinamento, che da lui trasse impulso, caratterizzò l'intero periodo aragonese, tanto da apparire come la naturale premessa della dominazione spagnola. 